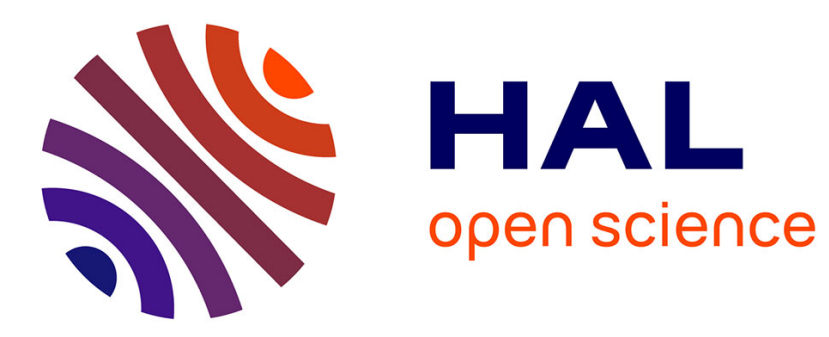

\title{
A VHDL-AMS model of RF MEMS capacitive shunt switches
}

Michael Kraemer, Daniela Dragomirescu, Vincent Puyal, Robert Plana

\section{To cite this version:}

Michael Kraemer, Daniela Dragomirescu, Vincent Puyal, Robert Plana. A VHDL-AMS model of RF MEMS capacitive shunt switches. International Semiconducteur Conference, CAS 2009, Oct 2009, Sinaia, Romania. hal-00449486

\section{HAL Id: hal-00449486 https://hal.science/hal-00449486}

Submitted on 21 Jan 2010

HAL is a multi-disciplinary open access archive for the deposit and dissemination of scientific research documents, whether they are published or not. The documents may come from teaching and research institutions in France or abroad, or from public or private research centers.
L'archive ouverte pluridisciplinaire HAL, est destinée au dépôt et à la diffusion de documents scientifiques de niveau recherche, publiés ou non, émanant des établissements d'enseignement et de recherche français ou étrangers, des laboratoires publics ou privés. 


\title{
A VHDL-AMS MODEL OF RF MEMS CAPACITIVE SHUNT SWITCHES
}

\author{
Michael Kraemer*,**, Daniela Dragomirescu*,**, Vincent Puyal,*,**, Robert Plana*,** \\ * CNRS ; LAAS ; 7 avenue du colonel Roche, F-31077 Toulouse, France \\ ** Université de Toulouse ; UPS, INSA, INP, ISAE ; LAAS ; F-31077 Toulouse, France \\ mkraemer@laas.fr, daniela@laas.fr,plana@laas.fr
}

\begin{abstract}
This paper is situated in the context of heterogeneous integration of millimeter-wave communication circuits. It shows that VHDL-AMS models are well suited for the simulation of building blocks constituting an integrated communication system. Simplicity and reduced execution time are mandatory when it comes to the simulation of the behavior of an entire SiP.

In particular, a VHDL-AMS model for RF MEMS capacitive shunt switches based on its equivalent circuit is presented. Subsequently, this model is, together with a transmission line model, applied to simulate the behavior of a mm-wave phase shifter employing RF MEMS shunt switches.
\end{abstract}

Keywords: SiP, SoC, RF MEMS, VHDL-AMS

\section{INTRODUCTION}

For future high-data rate communication systems the ultra-wide frequency band around $60 \mathrm{GHz}$ represents a promising alternative to the overcrowded spectra available at lower frequencies [1]. To avoid connection losses and achieve small system size and very low cost for high volume production, the transceivers employed in this frequency band will typically be designed as System on Chip (SoC) in a sub-micron silicon technology.

The limit for CMOS SoC integration usually is reached at the interface to the antenna. At $60 \mathrm{GHz}$, due to small size and high directivity of the antennas, phased array antennas are advantageous. However, their integration on a lossy silicon substrate of a standard CMOS processes results in low gain.

One possibility to address this issue is a heterogeneous approach, where different technologies are combined to a System in Package (SiP) as shown in Fig. 1. Thus, sub-micron CMOS technologies with high $f_{\mathrm{T}} / f_{\max }$ can be combined with phase shifters using low loss RF MEMS (radio frequency micro electromechanical systems) switches and highly efficient antennas. If high output power is required, the power amplifier (PA) at the transmitter can use a technology other than CMOS, which supports higher supply voltages and thus eases the design of PAs producing an output power in excess of $10 \mathrm{dBm}$.

A current research topic is the behavioral modeling of such entire systems. A hardware description language ideally suited for this task is VHDL-AMS because of its capability to describe analog and mixed signal systems [2].

While for the digital part, the VHDL code used in the design process can be directly employed, for the analog part behavioral models are not directly available. In addition to that, at millimeter wave frequencies coupling between different circuit components can become a major issue and needs also be taken into account for system level design.

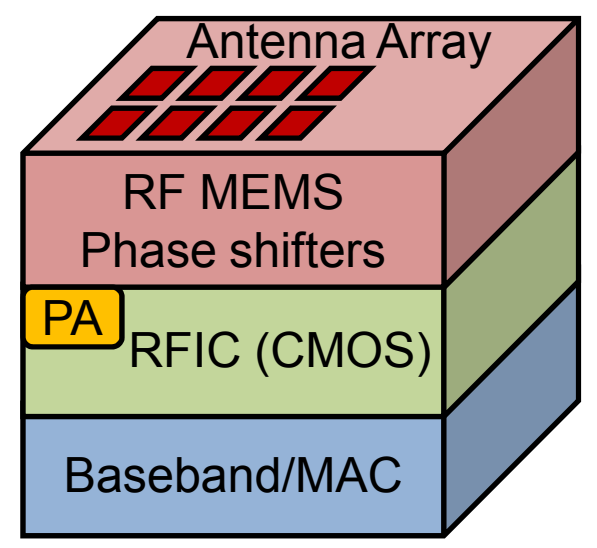

Fig. 1: SiP built by 3D integration of different technologies

The models of the different circuit blocks depend on the peculiarities of the respective circuits: For microwave $n$-ports like amplifiers, filters or mixers behavioral modeling techniques exist that represent the outputs of these components as a function of its current and past inputs. A modeling technique which employs artificial neural networks (ANNs) and lends itself to an implementation in VHDL-AMS is presented in [3].

A methodology to model the behavior of oscillators, which is not covered by modeling techniques for microwave n-ports, because the system outputs depending mainly on past system states rather that the system inputs, is presented in [4] by the authors of this article. It is very versatile, and can be applied to all kinds of oscillators, both single ended and differential, while being able to take into account voltage control. The model is derived from the circuit model used during design phase. It uses ANNs to approximate the non-linearity of the oscillator. A state space representation is employed to capture the non-linear dynamics of the oscillator, whose order is reduced substantially beforehand, assuring much faster execution than a circuit level model.

The behavior of new technologies, for example RF MEMS switches needs to be properly described as well. This paper presents an approach to use VHDLAMS to model RF MEMS capacitive shunt switches. 
It is based on an equivalent circuit model employing lumped elements and transmission lines as presented in [5]. Based on this model, a switched line phase shifter employing the RF MEMS capacitive switch as key building block is modeled to show the applicability of the RF MEMS model to complete circuit blocks.

\section{RF MEMS SWITCHES}

A die photo of a $60 \mathrm{GHz}$ RF MEMS capacitive shunt switch is shown in Figure 2. It consists of a metal bridge suspended over a coplanar waveguide (CPW), where the size of the bridge defines its capacitance and thus influences the frequency of operation.

An actuation voltage of around $30 \mathrm{~V}$, applied between the middle conductor of the CPW and the grounded bridge, causes the pull-in, i.e. the shortcircuiting of the signal path to ground by decreasing substantially the capacitance from its up-state value $C_{\text {up }}$ to its down-state value $C_{\text {down }}$. This is the behavior that has to be modeled in VHDL-AMS. The design and in-detail explication of this RF MEMS switch can be found in [6].

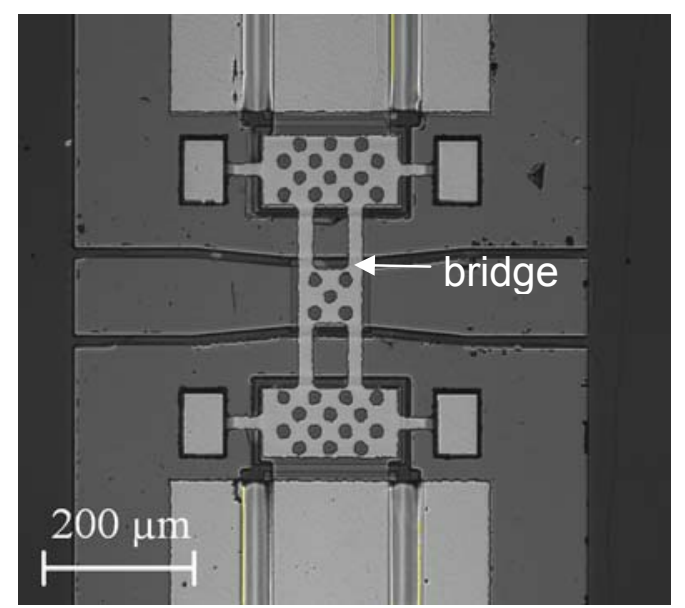

Fig. 2: Die photo of a $60 \mathrm{GHz}$ RF MEMS switch

The equivalent circuit model [5] of the RF MEMS capacitive shunt switch is given in Fig. 3. It consists of an RLC series resonance circuit connected by two short transmission lines. The only element that changes from up-state to down-state according to the switching signal $\mathrm{s}_{\mathrm{d}}$ is the capacitance $C$.

In [7], a scaling factor $K$ is introduced which allows to scale the dimensions of a reference device designed at $20 \mathrm{GHz}$ in order to obtain an RF MEMS switch working at any specified frequency up to $94 \mathrm{GHz}$. On the basis of this scaling factor, the parameters of the circuit model in Fig. 3 can be determined, thus allowing the generation of a scalable VHDL-AMS model useable over a large frequency range.

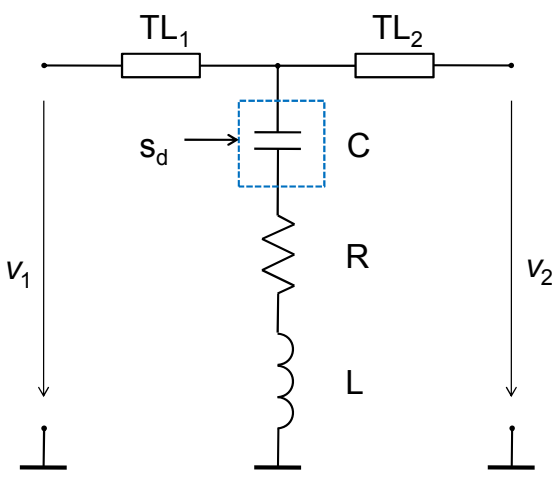

Fig. 3: RF MEMS switch equivalent circuit model

\section{THE VHDL-AMS MODEL}

The VHDL-AMS model contains two principal parts: The first part is the MEMS' resonating core, which uses a VHDL-AMS process to change the capacitance value according to the switching signal $s_{d}$. In order to simulate the switching time correctly, the two discrete capacitance values are mapped to a VHDL-AMS quantity using the keyword 'ramp. This keyword allows changing the capacitance value gradually from $C_{\text {down }}$ to $C_{\text {up }}$ during the specified time interval.

The equations describing the currents and voltages of the model core are implemented using simultaneous statements (indicated by $==$ ). The essential part of the associated VHDL-AMS code is:

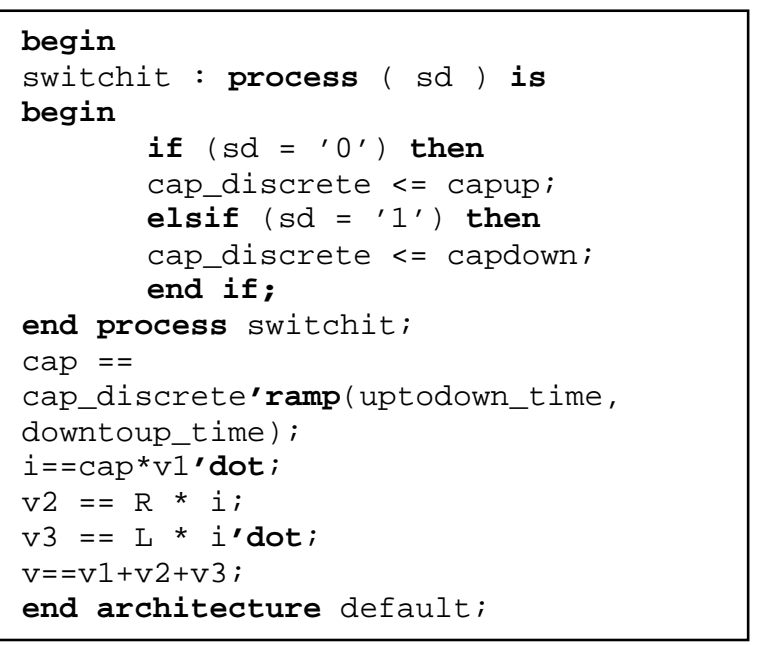

The second part is the transmission line (TL) model. It doesn't solve the line equations, but makes use of the fact that there is an incident wave and a reflected wave propagating on the line. The signal $V_{\text {to }}(t)$ is the incident voltage waveform at the beginning of the TL, while $V_{\text {back }}(t)$ is the reflected voltage waveform at the end of the TL. The associated current waveforms are calculated using the line's characteristic impedance $\mathrm{Z}_{0}$. To obtain the voltage waveforms at any other location on the TL, the signals $V_{\text {to }}(t)$ and $V_{\text {back }}(t)$ are delayed 
using the 'delayed statement of VHDL-AMS and specifying the wave velocity and the length of the line. To take into account loss, an exponential attenuation $\alpha$ is specified. Eventually, input and output voltages and currents are the superposition of these incident and reflected waves after experiencing the associated delay and attenuation.

The essential part of the TL's VHDL-AMS code is

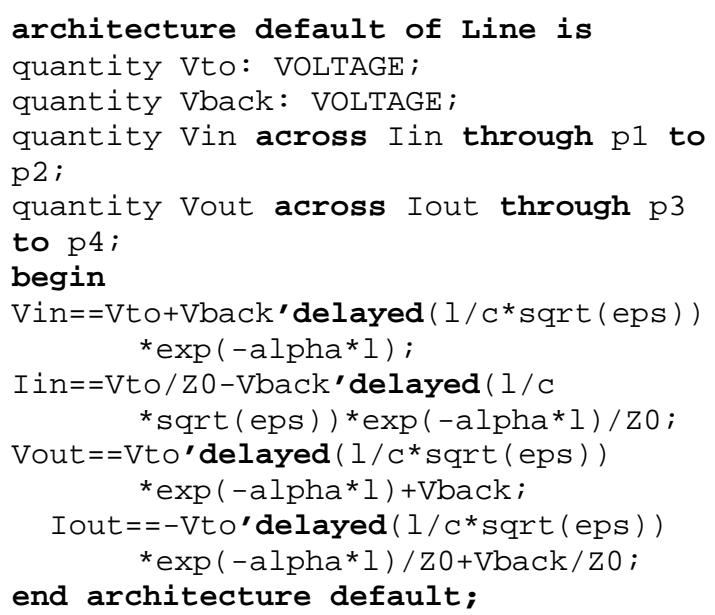

Note that the attenuation $\alpha$ used for modeling the loss on the TL is constant, and thus the same at all frequencies. Explicit frequency dependence, though physically present due to skin effect and dielectric loss, cannot be easily embodied in the suggested time domain model. However, a frequency domain model is not suitable, because the system simulation includes digital VHDL models that exclusively work in time domain.

In order to more correctly take into account loss, a transmission line model similar to the one implemented in [8] can be used. The partial differential equations describing the TL are represented by a ladder network with sufficient elements to account for the distributed nature of the TL. The VHDL-AMS simulator then solves these equations in time domain. While yielding a higher accuracy, the parameterization of this kind of model is more difficult, and the computation time increases considerably with respect to the suggested simple model, thus jeopardizing the effort to reduce complexity and computation time.

\section{RF MEMS MODEL RESULTS}

In Fig. 4 the insertion loss of the $60 \mathrm{GHz}$ RF MEMS switch in up-state is plotted. The general agreement between measurements and VHDL-AMS simulation is good. The variations of the insertion loss can be explained by the difficulty of measuring low loss transmission factors at these frequencies. In Fig. 5 the isolation is shown. Both resonance frequency and the associated strong attenuation of the signal are faithfully reproduced by the model. These results thus confirm the validity of using the TL model described in section 3 for the TLs contained in the RF MEMS equivalent circuit.

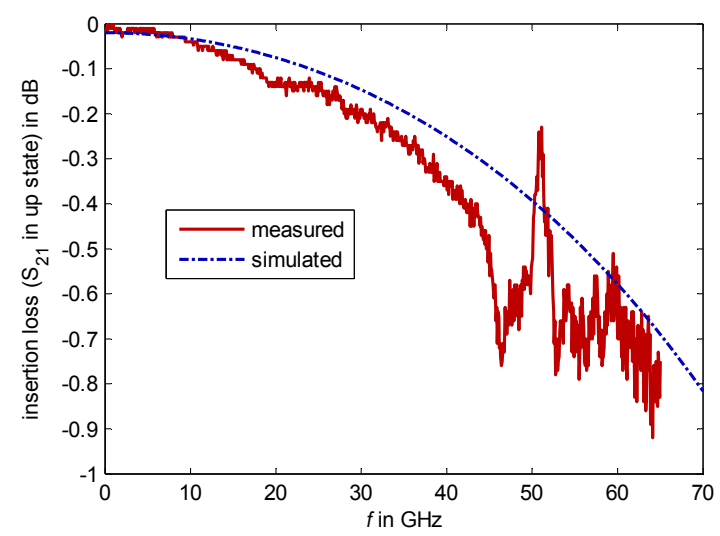

Fig. 4: Insertion loss in up-state of the $60 \mathrm{GHz} R F$ MEMS, measurements versus VHDL-AMS simulation

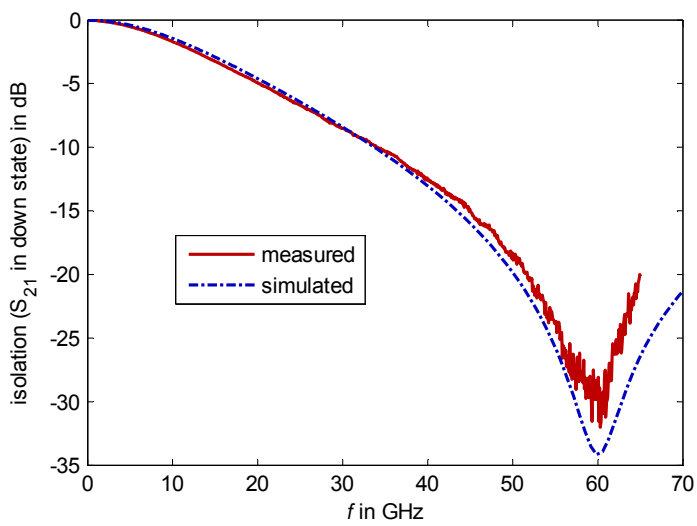

Fig. 5: Isolation in down-state of the $60 \mathrm{GHz} R F$ MEMS, measurements versus VHDL-AMS simulation

\section{APPLICATION OF THE MEMS MODEL TO PHASE SHIFTERS}

One application of RF MEMS switches in a heterogeneous communication system as described above is phase shifters. They are used to change the beam direction of antenna arrays.

The VHDL-AMS models developed in section 3 are employed in the following to the one bit SP2T switched line phase shifter presented in [9]. Figure 6 shows the schematic of this phase shifter. The outermost MEMS M1, M4, M5 and M8 are blocked in up-state and serve as capacitors for matching purposes. The switching signal ' $\mathrm{S}$ ' is used to actuate the MEMS M2, M3 and M6,M7 respectively, to commute between a long TL and a short TL, with the goal of attaining $45^{\circ}$ phase difference between the two paths. The TLs between the RF MEMS are used as interconnect and for matching purposes (refer to [9] for circuit details). 


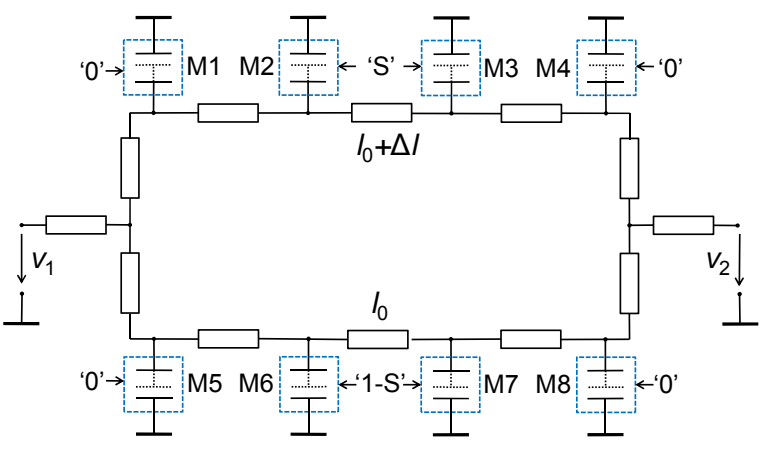

Fig. 6: schematic of switched line phase shifter composed of RF MEMS and transmission lines

A VHDL-AMS model of the phase shifter was created by generating a netlist connecting RF MEMS and TL models in order to obtain the structure of Fig. 6. Fig. 7 and Fig. 8 compare the simulation results of both VHDL-AMS and ADS model for the two states of the phase shifter.

Concerning insertion phase (Fig. 7), excellent agreement was achieved between both models. The relative phase shift at $60 \mathrm{GHz}$ is $44.93^{\circ}$ (VHDLAMS) versus $44.96^{\circ}$ (ADS).

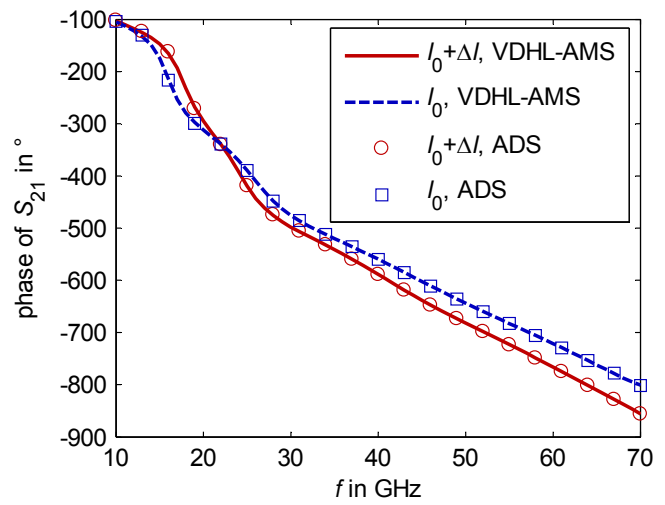

Fig. 7: Insertion phase shift for both states of the phase shifter, VHDL-AMS versus ADS

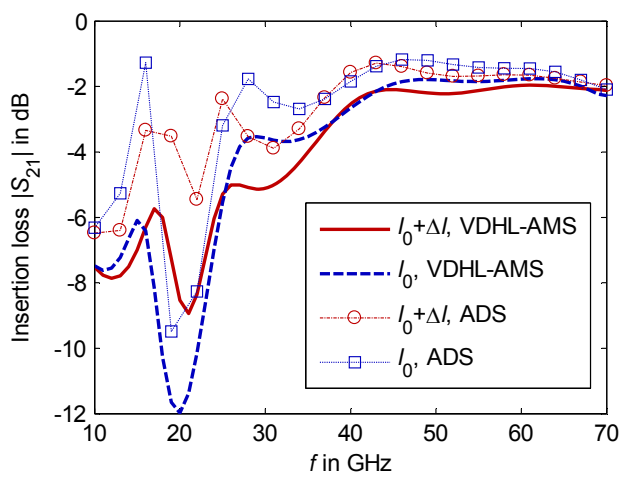

Fig. 8: Insertion loss for both states of the phase shifter, VHDL-AMS versus ADS

Concerning insertion loss (Fig. 8), the VHDL-AMS model's accuracy is very good in a large band around
$60 \mathrm{GHz}$, but decreases towards lower frequencies. This has two reasons: First, as mentioned in section 3, the TL models are only accurate at one frequency depending at the attenuation $\alpha$ (here at $60 \mathrm{GHz}$ ), while for lower frequencies loss is overestimated. Second, the VHDL-AMS model uses exactly the characteristic impedance $\mathrm{Z}_{0}$ specified, while in ADS the simulated value slightly differs from the specified one.

\section{CONCLUSION}

A simple and yet accurate RF MEMS shunt switch VHDL-AMS model, based on an equivalent circuit using lumped and distributed elements has been presented. Its performance was confirmed with respect to measurements.

The application of the model to simulate a switched line phase shifter showed excellent agreement in phase shift at all frequencies, while its insertion loss is accurately modeled in the operating band around 60 $\mathrm{GHz}$.

Together with models of other key building blocks like amplifiers, mixers, oscillators etc. the presented RF MEMS model can be used when creating behavioral models of whole SiPs. Future research will concentrate on the creation of models that represent the coupling between these blocks when integrated on the same chip or in the same package.

\section{REFERENCES}

[1] N. Guo, R.C. Qiu et al.: "60-GHz Millimeter-Wave Radio: Principle, Technology and New results", EURASIP Journal on Wireless Communications and Networking, 2007 [2] "IEEE standard VHDL analog and mixed-signal extensions: Std. 1076.1-1999," 1999.

[3] J. Wood, D. Root et al., "A behavioral modeling approach to nonlinear model-order reduction for RF/microwave ICs and systems," IEEE Trans. Microw. Th. \& Techn., Vol. 52, pp. 2274-2284, 2004.

[4] Kraemer, M., Dragomirescu, D., Plana, R.: “A Nonlinear Order-Reducing Behavioral Modeling Approach for Microwave Oscillators", IEEE Trans. on Microwave Theory and Techniques, 2009, Vol. 57, No. 2, pp. 991-1006

[5] Muldavin, J. \& Rebeiz, G. "High-isolation CPW MEMS shunt switches. 1. Modeling", IEEE Transactions on Microwave Theory and Techniques, 2000, Vol. 48, pp. 1045-1052

[6] V. Puyal, D. Dragomirescu et al. "RF MEMS Design for Phase Shifters from K-band up to W-band", Asia-Pacific Microwave Conference 2008, 16-20 Dec. 2008.

[7] Puyal, V.; Dragomirescu, D. \& Plana, R., "A new approach to RF MEMS shunt switch modeling from K-band up to W-band", Proceedings of SPIE. Advanced Topics in Optoelectronics, Microelectronics, and Nanotechnologies IV, 2009, 7297, pp. 729720-729720

[8] Nikitin, P. V. \& Shi, C. R., "VHDL-AMS based modeling and simulation of mixed-technology microsystems: a tutorial", Integration - The VLSI Journal, 2007, No. 40, pp. 261-273

[9] Puyal, V.; Dragomirescu, D.; et al. "Déphaseurs à base de MEMS RF à $60 \mathrm{GHz}$ pour systèmes à réseaux d'antennes reconfigurables", 16èmes Journées Nationales Microondes, Grenoble, 2009, p. 4F-10 